\title{
Desafios para gestão do trabalho a partir de experiências exitosas de expansão da Estratégia de Saúde da Família
}

\author{
Challenges for human resources management \\ from successful experiences of Family H ealth Strategy expansion
}

$M$ aria $\mathrm{H}$ elena $\mathrm{M}$ agalhães de $M$ endonça ${ }^{1}$

$M$ aria Inês Carsalade $M$ artins ${ }^{2}$

LigiaGiovanella ${ }^{1}$

Sarah Escorel ${ }^{1}$

${ }^{1}$ Departamento de Planejamento e Administração em Saúde, Escola Nacional deSaúde Pública Sergio Arouca,

Fundação Oswaldo Cruz. Rua Leopoldo Bulhões 1.480, Manguinhos. 21041-040 Rio deJaneiro RJ. mhelenam@ensp.fiocruz.br ${ }^{2}$ Departamento deCiências Sociais, Escola Nacional de Saúde Pública Sergio Arouca, Fundação Oswaldo Cruz.
Abstract This article discusses the management of the work in Family Health Strategy in four major urban centers. The research includes perspectives from different actors who compose and integrate the network of working relationships in Public Health System through questionnaires with employees of professional categories family health team and interviews with managers and representatives of professional bodies. It is a qualitative quantitativeevaluation study. The dimensions analysed were: insertion and remuneration policies, strategies and qualification of employees. The insertion and remuneration policy highlights the replacement of outsourced frames and hiring by public tender that allows links labor more stable. Other strategies are the establishment of allowance for expertise in areas of greater social vulnerability and the assimilation of specialists in Family and Community M edicinewith other experts engaged in secondary services. The political will of municipal $M$ anager to qualify the workforce of family health, maintaining the provision of adequate human resources needs of the health system is a fundamental factor for the consolidation of family health strategy in the face of the low degree of specialization of professionals to work in primary health care.

Key words M anagement of labor, Family health strategy, Qualification of professional of health primary care
Resumo Este artigo discute a gestão do trabalho na Estratégia deSaúde da Família em quatro grandescentros urbanos. A pesquisa contempla asperspectivas de diferentes atores que compõem e integram a rede de relações de trabalho no Sistema Único de Saúde por meio de questionários com trabalhadores das categorias profissionais da equipede saúde da família e entrevistas com gestorese representantes das entidades profi ssionais. Tratase de estudo de avaliação qualiquantitativo. A política de inserção e remuneração evidencia a substituição dos quadros tercei rizados e a contratação por concurso público, que possibilita vínculos trabalhistas mais estáveis. O utras estratégias são 0 estabelecimento de abono para atuação em áreas demai or vulnerabilidade social ea equiparação do salário dos médicos especialistas em medicina de família e comunidade com demais especialistas atuantes nos servi ços secundários. A vontade política do gestor municipal para qualificar a força de trabalho da saúde da família, mantendo a oferta de recursos humanos adequados às necessi dades do sistema de saúde, é fator fundamental para a consolidação da Estratégia de Saúde da Família, em face do baixo grau de especialização dos profissionais para atuar em atenção primária em saúde.

Palavras-chave Gestão do trabalho, Estratégia de Saúde da Família, Qualificação dos profissionais de saúde da atenção primária à saúde 
Introdução

A pesquisa “Implementação da Estratégia de Saúde da Família em Grandes Centros Urbanos", concluída em 2009, contempla as perspectivas de diferentes atores que compõem eintegram a rede derelações detrabal ho no Sistema Ú nico deSaúde (SUS), buscando uma visão global do processo de implementação da Estratégia de Saúde da Família (ESF), em um contexto de mudanças na organização do trabalho na esfera pública setorial. A pesquisa busca investigar o quanto o modelo de incorporação de pessoal pelos municípios está diretamente relacionado com as políticas de incorporação de pessoal do governo federal e como se explicitam em estratégias próprias de gestão do trabalho.

Por gestão do trabalho no setor público de saúde, entende-se o esforço do Estado, nosdiferentes níveis de governo, de regulação das relações sociais detrabalho, capacitação eformação profissional dos agentes sociais e criação de condições adequadas de trabalho. 0 conjunto desses fatores deve dar suporte a um processo de trabalho diferenciado, que determina mudança no modelo assistencial no sentido de ofertar uma atenção primária à saúde (APS) resolutiva e de qualidade e de uma ação voltada para o campo social.

A gestão do trabalho em saúde ganhou centralidade no processo de implementação da Estratégia de Saúde da Família na medida em quea adoção do novo modelo assistencial implica a ampliação e fixação do quadro de pessoal em nível municipal, num momento detransição econômica e de reforma de Estado que, contraditoriamente, tinha como diretriz a diminuição da partici pação do Estado na prestação de serviços.

Nos anos noventa, mais especificamente a partir de 1995, o plano de reforma administrativa estabeleceu novas normas jurídicas para o trabalho e permitiu que instituições privadas em associação com o Estado cumprissem seus obje tivos de bem-estar social. Para Nogueira ${ }^{1}$, a reforma efetuada pelo governo federal objetivou a redução do papel do Estado, a diminuição dos gastos públicos e favoreceu a desregulamentação das relações de trabalho no setor público. Para Cardoso J r. ${ }^{2}$, medidas "flexibilizadoras", criadas por dispositivos legais complementares, modificaram as condições do uso da força de trabaIho, de sua remuneração, de sua proteção, assim como suas estruturas de representação - sindicatos e Justiça do Trabalho.

No setor público de saúde, esta tendência de afastamento dos princípios que orientavam 0 trabalho na administração pública afetou os estados e municípios que, com a Constituição de 1988, passaram a ser responsáveis pela prestação de serviços de saúde na sua integralidade, necessitando para isto reestruturar eampliar seu quadro próprio de pessoal.

As medidas restritivas para contratação de pessoal no setor público, entre elas a Lei de Responsabilidade Fiscal, versus a demanda por recursos humanos resultou, nos estados e, sobretudo, nos municípios, na proliferação decontratos de trabalho pouco regulados, mediados por cooperativas, fundações privadas e ONGs, chamados de "contratos precários".

Nogueira ${ }^{1}$ chama a atenção para a especificidade do termo precariedade dos contratos quando se trata de trabalhadores no serviço público: Pode-se dizer que é irregular todo o vínculo de trabalho em que o trabalhador foi admitido na instituição pública sem concurso ou seleção de natureza pública, sendo exceção o trabalhador terceirizado admitido nas áreas não-finalísticas (como serviços gerais, segurança, mediante normas legais de licitação․ Para este autor, a precariedade do vínculo para uma ação finalística, como a prestação de serviços de saúde em estabelecimentos de saúde ou em atividades de saúde, se caracteriza quando o Estado não consegue fazer cumprir os requisitos constitucionais de concurso público (Constituição Federal, art. 37, incisos I ell) e de aplicação geral de um regime jurídico único detrabalho (Constituição Federal, art. 39).

A Estratégia de Saúde da Família, implementada no SU S neste período, foi uma das políticas setoriais fortemente impactada por este novo arranjo político-institucional, como demonstram diversos estudos que evidenciam um percentual elevado de trabal hadores de saúde com vínculos trabal histas através de entidades diversas ${ }^{1,3,4}$.

A Estratégia de Saúde da Família, ao se expandir para os grandes centros urbanos, onde encontrava uma estrutura de serviços mais consolidada, teve o desafio de combinar diversas estratégias de emprego, utilizando-se de diferentes vínculos trabalhistas 4 .

A elaboração de novo perfil profissional a partir da relação de trabalho e do processo de educação continuada dos profissionais não excluiu o aproveitamento do quadro estatutário das Secretaria M unicipal de Saúde(SM S), condicionado a que os profissionais aceitassem a alteração do processo de trabalho e a pactuação de alguma forma de complementação salarial na Estratégia de Saúde da Família no âmbito municipal. 
0 início da primeira década do século XXI segue a tendência dos anos noventa de combinar diversas estratégias para inserção profissional em face da implementação da Estratégia de Saúde da Família; contudo, mais recentemente, a mesma pode não ser confirmada, como se observa nos resultados da presente pesquisa.

Senum primeiro momento, entre 1995 a 2004, o governo federal restringiu os concursos públicos e estimulou outras formas de contratação, num segundo momento, de 2005 a 2008, estabeleceu como meta a regularização das relações de trabalho no setor público, com vistas à desprecarização do trabalho no mesmo. Hoje, já se evidenciam novos parâmetros para a gestão do trabalho, especialmente no nível municipal, quando os contratos "precários" estão sendo substituídos por quadros próprios através da realização de concursos públicos.

Outra variável importante que impactou 0 mercado de trabalho no setor saúde eque o diferencia do mercado de trabalho mais geral a ser levantada pela pesquisa é a particularidade do processo de trabal ho e da estrutura ocupacional no setor público de saúde. $\mathrm{Na}$ saúde, segundo Dedecca et al. ${ }^{5}$, existem funções eatividades diretamente relacionadas a determinadas categorias profissionais, que exigem uma qualificação própria e são reguladas externamente, com o controle do poder público.

Isso remete a uma terceira variável, que é a qualificação de recursos humanos, que se colo$\mathrm{ca}$, atualmente, na literatura sobre gestão, como um diferencial competitivo no mercado de trabal ho ecomo forma de garantir eficiência e eficácia às propostas e projetos institucionais.

As inserções de diferentes categorias profissionais no mercado de trabalho público e privado, tanto em sua dimensão ocupacional quanto em sua extensão, se refletem na Estratégia de Saúde da Família enquanto dificuldade dos municípios em contratar efixar os profissionais médicos das equipes da saúde da família e fazê- los cumprir a carga horária integral de quarenta horas semanais, já observada em outros estudos ${ }^{3}$.

$\mathrm{Na}$ área da saúde, os avanços da ciência e o desenvolvimento tecnológico acelerado, característicos do modo de produção atual, implicam a necessidade deuma constante atual ização de seus profissionais e uma base de conhecimentos interdisciplinar sólida que Ihes permitam enfrentar a diversidade e a "adversidade" do processo saúde no mundo atual. No caso específico da Estratégia de Saúde da Família, como modelo de organização da atenção, a necessidade de forma- ção técnica de núcleo edesenvolvimento dosprofissionais de forma permanente torna este desafio da qualificação ainda maior, pois questiona as formas tradicionais já consolidadas e socialmente reconhecidas de educação.

Assim, a qualificação surge na discussão sobre políticas de recursos humanos em saúde como componente que permite criar e manter a oferta de recursos humanos adequados às necessidades do sistema de saúde, debate que ganha força quando se considera o contexto de mudança na organização do sistema com a implantação e expansão da Estratégia de Saúde da Família.

O Ministério da Saúde, importante indutor da adoção dessa estratégia, vem enfrentando esse desafio em sua atual política de governo, com o reconhecimento e valorização do trabalho humano, através de políticas voltadas para este campo. Assim, em 2003, a criação da Secretaria de Gestão do Trabalho e da Educação na Saúde (SGTES) significou dar à gestão dos recursos humanosum tratamento estratégico, focando na dimensão do trabalho e da educação na saúde para a formulação de políticas orientadoras da gestão, formação, qualificação e regulação dos trabalhadores da saúde no Brasil ${ }^{6}$.

Dois programas vêm contribuindo direta e indiretamente para que a gestão do trabal ho no SUS se qualifique: (1) o Programa de Capacitação Gerencial para o Sistema Único de Saúde (PROGESUS), voltado para a qual ificação degestores, com vistas a enfatizar o importante papel dos gerentes dearticulador de ações que resultem na melhoria da qualidade de atenção, a partir da estruturação/qualificação dos serviços de saúdee (2) o programa da Universidade Aberta do Sistema Ú nico de Saúde (UNASUS), criado em junho de2008, quefacilita o funcionamento deuma rede colaborativa de instituições acadêmicas, serviços de saúde e gestão do SUS, destinada a atender necessidades de formação e educação permanente em saúde em áreas estratégicas?.

Em 2003, também foi aprovada a residência em medicina da família e da comunidade, destinada a formar médicos especialistas em atenção primária, com enfoque comunitário, para atuarem na perspectiva da promoção de saúde. Inicia-se, portanto, um novo modelo de residência médica, cujo lócus de atuação foge ao modelo tradicional, que se localiza no sistema hospitalar.

A estratégia de residência como forma de qualificação e especialização dos profissionais de saúde vem se estendendo para as demais profissões de saúde, na modalidade de cursos de especialização nos moldes de residência, com o obje- 
tivo de qualificar os profissionais de saúde para trabalhar na Estratégia de Saúde da Família.

Esta éuma resi dência orientada pelos princípios ediretrizes do SUS, a partir das necessidades e realidades locais e regionais, e abrange as profissões da área da saúde, a saber: biomedicina, ciências biológicas, educação física, enfermagem, farmácia, fisioterapia, fonoaudiologia, medicina veterinária, nutrição, odontologia, psicologia, serviço social e terapia ocupacional (Resolução CN S no 287/1998). Estas práticas podem ser observadas nas realidades estudadas.

0 atual artigo analisa e discute, com base no contexto acima exposto, as modalidades de gestão do trabalho em saúde em cada município estudado, consolidando dados que permitem 0 conhecimento do perfil dos profissionais das equipes de saúde da família quanto a sexo, idade, formação básica e pós-graduada, experiência anterior de trabalho; da política de inserção e fixação dos profissionais das equipes de saúde da família na estrutura organizacional da SM S e da política de qualificação para a atuação na Estratégia de Saúde da Família.

\section{M etodologia}

Este artigo discute parte dos resultados de pesquisa que consistiu no estudo de quatro casos da implementação da Estratégia de Saúdeda Família em grandes centros urbanos. A escolha dos municípios se deu de forma intencional, por serem considerados experiências consolidadas deimplementação da Estratégia de Saúde da Família por apresentar tempo mínimo deimplantação demais de seis anos, elevada cobertura da Estratégia de Saúde da Família, expansão das equipes de saúde da família e inovações no modelo assistencial.

Os municípios estudados são Aracaju, Belo Horizonte, Florianópolis e Vitória. Os estudos de caso realizados correspondem a uma estraté gia de pesquisa al icerçada em metodologias quantitativa e qualitativa e diversas fontes de informação convergentes, que foram trianguladas para responder às perguntas da investigação ${ }^{8}$.

$N$ as quatro cidades, foram levantadas informações de três fontes: gerentes municipais com realização de entrevistas (77), profissionais das equipes de saúdeda família com questionários autoaplicados (1.336) efamílias cadastradas com aplicação de questionários estruturados nos domicílios ( 3.312 famílias). Neste artigo, são anal isadas as informações dos gerentes e dos profissionais de nível superior das equipes de saúde da família.
Para a análise da gestão do trabalho, escoIheu-se três dimensões: (1) política municipal de inserção dos profissionais como formas de absorção dos profissionais da rede, captação deprofissionais para a estratégia, remuneração, criação de benefícios ou incentivos, forma de contrato e vínculo institucional; (2) estratégias defixação de profissionais de saúde e rotatividade dos profissionaise (3) política municipal de qualificação.

A pesquisa foi financiada pelo $D$ epartamento deAtenção Básica da Secretaria deA tenção à Saúde do M inistério da Saúde, com recursos do Fundo Nacional de Saúde.

\section{Resultadosediscussão}

\section{Inserção e remuneração}

A análise do perfil dos profissionais das equipes de saúde da família aponta para uma forte feminilização da força de trabal ho e para a presença de mais deum terço dos profissionais adultos jovens entre os médicos e enfermeiros nas equipes, como pode ser observado no Quadro 1. Observou-se, ainda neste quadro, um percentual elevado de profissionais, com experiência anterior na Estratégia de Saúde da Família, aproximadamente $80 \%$ no caso dos médicos, com exceção de Belo Horizonte. Também um terço dos médicos e quase $50 \%$ dos enfermeiros (exceto em Florianópolis) já apresentavam experiência anterior de trabal ho na SM S.

Associando este perfil de jovens adultos com 0 fato de grande parte destes profissionais, sobretudo os médicos, terem experiência anterior de trabalho na Saúde da Família, é possível inferir que a Estratégia de Saúde da Família pode estar se constituindo como um campo de trabaIho atraente para estes profissionais.

A menor adesão das enfermeiras, com ênfase para o caso de Florianópolis, sugere a necessidade de pensar estratégia específica para esses profissionais 9 .

A análise do processo de inserção apresentada no Quadro 2 indica que, atualmente, mais de $90 \%$ de médicos e enfermeiros são contratados pela SM S. Esta situação caracteriza uma reversão no quadro de multiplicidade de vínculos e muitas vezes até de ausência dos mesmos, que marcou a fase de implantação da Estratégia de Saúde da Família $a^{3,4}$.

A realização de concursos públicos para substituição dos quadros terceirizados em todas as categorias profissionais tem sido uma preocu- 
Quadro 1. Perfil dos profissionais de nível superior das equipes de saúde da família em quatro centros urbanos, 2008.

\begin{tabular}{|c|c|c|c|c|}
\hline Características & Aracaju & Belo Horizonte & Florianópolis & Vitória \\
\hline $\begin{array}{l}\text { Sexo } \\
\% \text { médicos sexo feminino } \\
\% \text { enfermeiros sexo feminino }\end{array}$ & $\begin{array}{l}70,0 \\
89,0\end{array}$ & $\begin{array}{l}58,0 \\
92,0\end{array}$ & $\begin{array}{l}56,0 \\
91,0\end{array}$ & $\begin{array}{l}77,0 \\
90,0\end{array}$ \\
\hline $\begin{array}{l}\text { Faixa etária } \\
\text { \% médicos entre } 31 \text { e } 40 \text { anos } \\
\% \text { enfermeiros entre } 31 \text { e } 40 \text { anos }\end{array}$ & $\begin{array}{l}21,0 \\
27,0\end{array}$ & $\begin{array}{l}40,0 \\
33,0\end{array}$ & $\begin{array}{l}34,0 \\
23,0\end{array}$ & $\begin{array}{l}43,0 \\
32,0\end{array}$ \\
\hline $\begin{array}{l}\text { Experiência de trabalho anterior em SF } \\
\% \text { médicos com experiência anterior em SF } \\
\% \text { enfermeiros com experiência anterior em SF }\end{array}$ & $\begin{array}{l}81,0 \\
73,0\end{array}$ & $\begin{array}{l}69,0 \\
61,0\end{array}$ & $\begin{array}{l}85,0 \\
49,0\end{array}$ & $\begin{array}{l}80,0 \\
68,0\end{array}$ \\
\hline $\begin{array}{l}\text { Experiência de trabalho na SM S } \\
\% \text { médicos já atuantes na SMS } \\
\% \text { enfermeiros já atuantes na SMS }\end{array}$ & $\begin{array}{l}32,0 \\
47,0\end{array}$ & $\begin{array}{l}31,0 \\
48,0\end{array}$ & $\begin{array}{l}31,0 \\
19,0\end{array}$ & $\begin{array}{l}26,0 \\
48,0\end{array}$ \\
\hline
\end{tabular}

Fonte: NU PES/DAPS/EN SP/Fiocruz - Pesquisa Saúde da Família: quatro estudos decaso, 2008.

M édicos em Aracaju ( $N=56)$ BH ( $N=72)$; Florianópolis $(N=61)$; Vitória $(N=35)$.

Enfermeiras em Aracaju ( $N=66)$; BH ( $N=75)$; Florianópolis ( $=70)$; Vitória $(N=50)$.

Quadro 2. Inserção dos profissionais das equipes de saúde da família na estrutura organizacional das SM S, em quatro centros urbanos, 2008.

\begin{tabular}{|l|c|c|c|c|}
\hline \multicolumn{1}{|c|}{ Inserção dos profissionais } & Aracaju & Belo Horizonte & Florianópolis & Vitória \\
\hline Instituição contratante & & & & \\
\% médicos vínculo SM S & 100,0 & 100,0 & 97,0 & 100,0 \\
\% enfermeiros vínculo SM S & 95,0 & 100,0 & 96,0 & 96,0 \\
\hline Forma de vinculação & & & & \\
\% médicos estatutários & 93,0 & 81,0 & 66,0 & 91,0 \\
\% enfermeiros estatutários & 92,0 & 95,0 & 81,0 & 100,0 \\
\hline
\end{tabular}

Fonte: NUPES/DAPS/EN SP/Fiocruz - Pesquisa Saúde da Família: quatro estudos de caso, 2008.

M édicos em Aracaju ( $N=56)$ BH ( $N=72)$; Florianópolis $(N=61)$; Vitória $(N=35)$.

Enfermeiras em Aracaju $(N=66) ; B H(N=75)$; Florianópolis $(N=70)$; Vitória $(N=50)$.

pação recorrente dos gestores da saúde dos municípios estudados. Essa forma de contratação tem possibilitado vínculos trabal histas mais estáveis, com impacto positivo na fixação dos profissionais na Estratégia de Saúde da Família, ao mesmo tempo em que encerra a perspectiva de melhoria de desempenho das ações de saúde.

0 Quadro 2 contempla ainda, na apresentação da inserção dos profissionais, as formas de vínculo mais praticadas nos municípios estudados e, assim, é possível observar o reflexo da política de "desprecarização do trabalho" quando são registrados como predominantes os vínculos estatutários entre os profissionais médicos e enfermeiros, em torno de $80 \%$ em quase todos os municípios estudados, à exceção dos médicos em Florianópolis (66\%).

$\mathrm{N}$ a gestão de recursos humanos para a atenção primária, a contratação por concurso público coloca-se como uma estratégia essencial, para facilitar a fixação de profissionais, embora não suficiente para manter os profissionais em suas posições de trabalho. Um fator limitante éa não vinculação desta estratégia à definição de plano de carreira específico para a área da saúde.

As entrevistas com gestores e a análise dos questionários com os profissionais evidenciam a permanência de dificuldades para atração e re- 
tenção dos profissionais médicos, sobretudo de vido à carga horária de quarenta horas semanais, associada à exigência, por parte de al gumas SM S, de dedicação exclusiva destes profissionais. Ademais, deve-se levar em consideração o apelo da prática liberal da medicina (ainda que simbólico) como concorrente à adesão desses profissionais à Estratégia de Saúde da Família.

Em um dos municípios, foi colocado por profissionais e representantes de entidades profissionais, como uma questão polêmica, a contratação dos profissionais das equipes de saúde da família em regime estatutário apenas por vinte horas, com extensão dejornada de trabal ho para as outras vinte horas, sem agregar todos os direitos trabal histas como aqueles relativos à aposentadoria.

Já a possibilidade de vínculo adicional com a SM S para realização de plantão em serviços de pronto-atendimento/urgência pelos profissionais médicos das equipes de saúde da família é apontada como um elemento facilitador na fixação dos profissionais (ao oferecer ganhos salariais e diversificação de ações assistenciais), podendo proporcionar melhora da articulação entre serviços de emergência e de atenção primária à saúde. Vale destacar que experiências européias indicam melhora de atendimento nas emergências com a contratação de especialistas em medicina de família e comunidade ${ }^{10}$.

De acordo com profissionais e gestores pesquisados, a efetivação dos quadros de pessoal das equipes de saúde da família ainda esbarra em dificuldades, destacando-se referências de baixa remuneração, especialmente entre os mé dicos diante da complexidade de suas atribuições face às condições sociais de trabalho. A pontam para a necessidade de aperfeiçoamento das habilidades e competências para lidar com 0 amplo contingente de problemas de saúde e as diversidades socioculturais das comunidades. Segundo eles, a atuação em áreas de maior vulnerabilidade social tensiona o trabal ho das equipes, que referem o estresse entre os profissionais como fator de abandono das funções.

Por fim, as iniciativas de equiparação salarial entre todos os profissionais de nível superior, utilizadas em alguns municípios, se apresentaram como insustentáveis, em médio prazo, considerando a estrutura de emprego, o mercado de trabalho e a oferta de profissionais qualificados. No caso de Aracaju, por exemplo, a isonomia conquistada pelos profissionais de saúde através deum plano de carreiras, na prática, permitiu de um lado que os profissionais não médicos aferissem salários com valores por vezes acima do mercado, garantindo sua adesão, mas, por outro, implicou o rebaixamento dos salários dos médicos, interferindo fortementeem sua adesão.

\section{Estratégias de fixação}

A fixação dos profissionais nas equipes de saúde da família foi outro fator analisado pela pesquisa e constitui-se ainda num desafio a ser enfrentado, emboraseobserveum índicedepermanência maior em relação ao início da Estraté gia de Saúde da Família.

No Quadro 3, considerando-se como parâmetro de fixação a permanência dos profissionais há mais de quatro anos na equipe da saúde da família, os resultados apontam um percentual significativo de permanência de profissionais (40 a $60 \%$ dos médicos e 48 a $68 \%$ dos enfermeiros) em três dos quatro municípios pesquisados. Estes resultados são atribuídos pelos gestores à regularização dos vínculos e às estratégias dequalificação. Ainda assim, um quinto ou mais

Quadro 3. Fixação dos profissionais das equipes da saúde da família, nos quatro centros urbanos, 2008.

\begin{tabular}{|l|c|c|c|c|}
\hline \multicolumn{1}{|c|}{ Tempo de atuação na ESF } & Aracaju & Belo H orizonte & Florianópolis & Vitória \\
\hline \% médicos até 12 meses & 7,0 & 18,0 & 23,0 & 23,0 \\
\hline$\%$ médicos + de 4 anos & 62,0 & 44,0 & 29,0 & 42,0 \\
\hline$\%$ enfermeiros até 12 meses & 9,0 & 16,0 & 48,0 & 32,0 \\
\hline \% enfermeiros + de 4 anos & 68,0 & 56,0 & 24,0 & 48,0 \\
\hline
\end{tabular}

Fonte: NU PES/DAPS/ENSP/Fiocruz - Pesquisa Saúde da Família: quatro estudos de caso, 2008.

M édicos em Aracaju ( $\mathrm{N}=56)$; $\mathrm{BH}(\mathrm{N}=72)$; Florianópolis ( $\mathrm{N}=61)$; Vitória $(\mathrm{N}=35)$.

Enfermeiras em Aracaju $(N=66) ; B H(N=75)$; Florianópolis $(N=70)$; Vitória $(N=50)$. 
dos profissionais atuavam na Estratégia de Saúde da Família do município a menos de doze meses, com exceção de Aracaju.

A rotatividade, no entanto, ainda constituise num entrave ao desenvolvimento do trabalho eéatribuída pelos gestores e profissionais a fatores diversos, tais como a remuneração aquém das expectativas, condições de trabalho, exigência da carga horária integral, baixa oportunidade de progressão profissional, baixo comprometimento dos profissionais, ausência de formação prévia compatível com o modelo proposto pela Estratégia de Saúde da Família9,11-13.

Uma das estratégias para a fixação e adesão dos médicos relatada pelos gestores de alguns municípios foi a equiparação do salário dos mé dicos especialistas em medicina de família e comunidade com os demais especialistas médicos atuantes nos serviços secundários.

Outra estratégia utilizada foi o estabelecimento de incentivos/complementação salarial para os profissionais das unidades de saúde da família (USF) escalonados segundo critérios de risco e vulnerabilidade das comunidades atendidas.

Gestores e profissionais apontam ainda a necessidade de outras interven ções de caráter gerencial nas USF, que promovam condições detrabalho adequadas ao desempenho das funções, tais como a boa organização, dependências físicas confortáveis e limpas, material apropriado e disponível, segurança física e ambiental.

Política de qualificação

A terceira dimensão considerada nesta análise foi a política municipal de qualificação dos profissionais, elemento crítico no processo de implementação da Estratégia de Saúde da Família no município. A qualificação dos profissionais de nível superior para a concretização da atenção primária à saúde no SUS é um grande desafio para a gestão pública e demanda estratégias de desenvolvimento profissional contínuo, de forma a aprimorar/desenvolver competências técnicas específicas de cada profissão ecompe tências do "campo" da atenção primária para ações coletivas e atuação comunitária.

O Quadro 4 apresenta indicadores de qual ificação dosprofissionais denível superior das equipes de saúde da família e mostra uma tendência de investimento na formação dos profissionais das equipes de saúde da família em nível local.

Os resultados expressam diversidade entre os municípios e entre as categorias profissionais em relação à qualificação dos trabalhadores de nível superior no que se refereà realização de cursos de especialização em saúdeda família, o quetem levado a uma busca por parte dos gestores por novos modelos de qualificação em parceria com centros formadores, universidades, escolas de saúde.

Em Florianópolis e Vitória, encontram-se os maiores percentuais de médicos com al guma pósgraduação, $85 \%$ e $77 \%$, respectivamente, sendo que em Florianópolis há presença de $44 \%$ de médi cos com especialização em Saúde da Família (SF) e 44\% de médicos com título de especialista em medicina defamília ecomunidade. Esses percentuais são bem mais baixos para os enfermeiros. Em Belo Horizonte, a qualificação especialmente quanto à especialização em SF dos médi$\cos (56 \%)$ e enfermeiros ( $76 \%)$ também é el evada. Contudo, a presença de médicos com título

Quadro 4. Qualificação dos profissionais das equipes de saúde da família em quatro centros urbanos, 2008.

\begin{tabular}{|l|c|c|c|c|}
\hline \multicolumn{1}{|c|}{ Formação } & Aracaju & Belo Horizonte & Florianópolis & Vitória \\
\hline \% médicos com alguma pós-graduação & 63,0 & 65,0 & 85,0 & 77,0 \\
\hline \% enfermeiros com alguma pós-graduação & 77,0 & 81,0 & 44,0 & 29,0 \\
\hline \% médicos com curso de especialização em SF & 16,0 & 66,0 & 44,0 & 29,0 \\
\hline $\begin{array}{l}\text { \% enfermeiros com curso de especialização } \\
\text { em SF }\end{array}$ & 39,0 & 76,0 & 21,0 & 36,0 \\
\hline $\begin{array}{l}\text { \% médicos com titulo de especialista } \\
\text { em medicina de família e comunidade }\end{array}$ & 5,0 & 28,0 & 44,0 & 9,0 \\
\hline
\end{tabular}

Fonte: N UPES/DAPS/EN SP/Fiocruz - Pesquisa Saúde da Família quatro estudos de caso, 2008.

M édicos em Aracaju ( $\mathrm{N}=56) ; \mathrm{BH}(\mathrm{N}=72)$; Florianópolis $(\mathrm{N}=61)$; Vitória $(\mathrm{N}=35)$.

Enfermeiras em Aracaju ( $N=66) ; B H(N=75)$; Florianópolis $(N=70)$; Vitória $(N=50)$. 
de especialista em medicina defamília ecomunidadeé de28\%.

No Brasil, segundo Machado et al. ${ }^{14}$, a cate goria médica obtém uma especialização basicamente através de dois mecanismos: o exame de ordem realizado por sociedades de especialistase as residências médicas, que exigem do residente tempo integral por um período de dois anos ou mais. 0 programa de residência médica constitui uma modalidade de ensino de pós-graduação destinada a médicos, funcionando em instituições de saúde, sob a orientação de profissionais médicos de el evada qualificação ética e profissional, sendo considerada o "padrão ouro" da especialização médica. E confere ao médico residente o título de especialista.

No entanto, a compatibilização entre a formação médica recebida ea atuação na Estratégia de Saúde da Família é discutida por al guns autores. Seixas e Stell $a^{15}$ e Padilha ${ }^{16}$ destacam que a formação médica pós-graduada está centrada em especialidades e subespecialidades, voltadas para ações de média e alta complexidade, reforçando a carência por profissionais com competências para desenvolver ações voltadas à proteção e à promoção da saúde. Destacam, ainda, que a formação em medicina ainda é tradicionalmente organizada segundo disciplinas desarticuladas, ciclos básico e clínico separados e centrados na doença e no atendimento hospitalar.

Assim, cria-se uma divergência entre as expectativas do profissional e o queéesperado dele, tendo em vista o modelo assistencial da Estraté gia de Saúde da Família. Há entre os médicos algum consenso de que a formação pós-graduada e o contato com a tecnologia mais avançada na área possibilitam ascensão a uma forma diferenciada deinserção profissional, associada a um padrão de vida elevado e a fixação em grandes centros urbanos, onde se encontram concentradas possibilidades de formação, aperfeiçoamento profissional, tecnologias e acesso a uma socie dade de consumo ${ }^{15,17}$.

O Curso de Especialização em Saúde da Família é uma das estratégias adotadas pela UNASU S ${ }^{6}$. A avaliação do Observatório de Recursos H umanos do IM S/U ERJ, entre2004 e2008, aponta que incentivos como os dados às especializações eresidências multiprofissionaissão uma opção para remodelar as práticas profissionais de forma mais rápida, para atender às demandas da organização dos serviços nos moldes da Estratégia de Saúde da Família7. Observa-se que as instituições das capitais pesqui sadas neste estudo (Belo H orizonte, Aracajú, Vitória, Florianópolis) foram contem- pladas com repasses de recursos para este fim. Tal visão de mundo impacta a adesão dos profissionais ao trabalho na Estratégia de Saúde da Família.

A preocupação com a garantia das competências ehabilidades-chavedos profissionais para cumprir os objetivos definidos para a reorganização da atenção primária à saúde é analisada por Pierantoni et al. ${ }^{18}$ e Souza et al. ${ }^{19}$, que afirmam que profissionais com estas novas competências são "profissionais que não estão disponíveis no mercado de trabalho, porque tradicionalmente foram formados e preparados para atuar no modelo tradicional".

O fato da SM S deFlorianópolis exigir o título deespecialista em medicina defamília ecomunidade como requisito ao ingresso dos médicos, via concurso público, ao quadro permanente da SM S, pode ser avaliada como um ponto positivo não só à adequação do perfil profissional dos médicos à Estratégia de Saúde da Família, mas também como fator de motivação para os médicos a permanecerem nesta área, constituindo-se em um indicador adicional deadesão desses profissionais à Estratégia de Saúde da Família.

Contudo, as iniciativas de oferta de formação são bem diferenciadas. Em alguns municípios, partem da gestão local (Belo H orizonte e $\mathrm{Vi-}$ tória) ${ }^{11,13}$; em outros, se caracterizam como um programa de extensão da universidade (Florianópolis) ${ }^{9}$. Destaca-se o caso de Aracaju ${ }^{12}$, que apresenta baixa oferta de formação de médicose enfermeiros e também de cursos de especialização eo efeito destebaixo investimento social pode ser observado nos resultados apresentados.

É possível notar um percentual maior deprofissionais qualificados na Estratégia de Saúde da Família naqueles municípios que têm um programa de desenvolvimento de recursos humanos estruturado. N este contexto, ressalta-se a necessidade de uma formação alinhada com os princípios ordenadores da Estratégia de Saúde da Família para o comprometimento dos profissionais, sobretudo médicos, com o modelo de atenção primária à saúde a ser implantado.

0 desenvolvimento de cursos de especialização em Saúde da Família pela própria SM S, em parceria com instituições acadêmicas em escala, têm permitido a qualificação em serviço efacilitado a adequação do perfil profissional para Estraté gia deSaúdeda Família em menor espaço detempo, como éo caso do município deBelo H orizonte e mais recentemente do município de Vitória.

A constituição das USF como campo de formação da graduação qualifica os serviços pres- 
tados pela Estratégia de Saúde da Família, como indicam os gestores. Destaque deve ser dado à integração promovida pela rede docente-assistencial, envolvendo a SM S de Florianópolis e a Universidade Federal de Santa Catarina, na qual as unidades de atenção primária à saúde básica constituem campo de formação de alunos dos diversos cursos de graduação da área da saúde e pós-graduação na modalidade resi dência multiprofissional em Saúde da Família.

Tal integração ainda possibilita aos profissionais que cursam residência em Saúde da Família ser atraídos para ingressar na Estratégia de Saúde da Família, embora não seja elemento suficiente para fixá-los. Por outro lado, a contribuição dessa integração para a capacitação continuada e educação permanente das equipes de saúde da família, no entanto, não está bem explicitada e poderia ser incentivada.

A qualificação dos profissionais das equipes de saúde da família e as estratégias de educação continuada com participação de profissionais das diversas modalidades de serviços (USF, atenção especializada, UPA) têm contribuído também para aumentar a credibilidade dos profissionais da atenção primária à saúde, superar relações hierárquicas eo isolamento entreatenção primária à saúde e especializada, segundo seus gestores.

Vale destacar que a qualificação técnica em APS éuma necessidade sentida pelos médicos em todos os municípios estudados, que demandam capacitações técnicas, clínicas, dirigidas especificamente à categoria. Esta demanda está fundamentada no argumento de que a competência técnicaé uma das bases da resolutividadeecredibilidade dos profissionais de atenção primária à saúde ${ }^{16}$. Assim, os gestores ressaltam que, para os médicos, uma formação específica poderia contribuir para reduzir os encaminhamentos aos especialistas, ao garantir maior segurança aos médicos quanto aos diagnósticos eà terapêutica.

Outro exemplo são as estratégias de qualificação em saúde pública dos gerentes das USF, que vêm propiciando melhor articulação deações coletivas e individuais na unidade básica. N este sentido, a incorporação ou a formação de sanitaristas por meio de curso de especialização pode contribuir para potencializar a vigilância em saúde com monitoramento de indicadores epidemiológicos de cada território em conjunto com as equipes.

Por fim, estratégias de educação permanente edesenvolvimento profissional contínuo com uso de tecnologias de informação e comunicação (TIC), como telessaúde (teleenfermagem, tele saúde bucal, telemedicina), em Belo H orizonte, tem facilitado a qualificação dos profissionais para o exercício de suas funções específicas e para o uso de diretrizes assistenciais, além de contribuir para a melhora da resolutividade da USF e promover a comunicação entre especialistas e generalistas, facilitando referências e coordenação dos cuidados pela atenção primária à saúde.

Em relação às estratégias de desenvolvimento de recursos humanos na APS com participação de profissionais de outros níveis de atenção, que promovem maior integração da atenção, observaram-se experiências e estágios distintos entre os municípios estudados.

Em Florianópolis, a implantação dos $N$ úcleos deA poio à EstratégiadeSaúdeda Família (NASF), por exemplo, colocava-se como uma prioridade na organização e qualificação dos serviços e dos trabalhadores.

Em Aracaju, num primeiro momento, a ênfase foi colocada no processo de educação permanente, quecompreendia a organização deprocessos pedagógicos com etapas de desenvolvimento do aprendizado que incluíam a problematização de situações concretas do cotidiano da assistência sob a orientação de agentes educacionais; contudo, essa experiência, segundo os gestores, foi interrompida em 2007.

Em Vitória, a consolidação do modelo assistencial está sendo pautada pelo modelo das "Iinhas da vida" e vem sendo operacionalizada através das rodas de educação permanente.

\section{Consideraçõesfinais}

Os recursos humanos em saúde constituem, reconhecidamente, um dos principais desafios ao processo deimplementação do Sistemaú nico de Saúde brasileiro. Tais desafios são ainda maiores quando se analisa a Estratégia de Saúde da Família, seja no que diz respeito à formação e qualificação dos profissionais para atuarem no âmbito das equipes de saúde da família, seja pela questão dos víncul os trabal histas e estratégias de fixação de pessoal, seja ainda pela adesão desses profissionais à proposta da Estratégia de Saúde da Família.

A gestão de trabalho no contexto da Estratégia de Saúde da Família nas cidades estudadas tem buscado equacionar dificuldades relativas à implementação do SUS no nível local. O que é evidenciado pela busca da regularização dos vínculos através da realização de concursos públicos, pelo investimento e valorização do processo de qualificação dos trabalhadores, pelo tempo 
de permanência dos profissionais nas equipes mais elevado, pela adoção de mecanismos deremuneração mais adequados por categoria e complementações via recompensas específicas para atuação em áreas de risco.

A vontade política do gestor municipal para formar a força de trabalho da saúde da família com vistas à sua fixação equalificação para a atenção primária à saúde é fator explicativo para diferenças de resultados entre as cidades quanto à formação voltada para a saúde da família. Ampliouse a consciência que profissionais inexperientes e sem formação em saúde pública, em medicina de família ecomunidadeou em saúde da famílialimitam 0 alcance das ações e a resolutividade da APS.

Em síntese, a análi ise dos resultados da pesquisa no processo de implementação da Estratégia de Saúde da Família nos municípios aponta para os seguintes aspectos que devem ser val orizadose destacados quanto à gestão do trabalho para a Estratégia de Saúde da Família: (1) a centralidade da gestão do trabalho e o investimento nos processos de qualificação, como prioridades da gestão; (2) as estratégias de educação permanente, que privilegiam o espaço de trabalho como de aprendizagem; (3) a existência de quadro permanentedeprofissionaisselecionados através deconcurso público; (4) a implantação de um sistema de remuneração diferenciada, deforma a incentivar e, ao mesmo tempo, remunerar o exercício profissional em locais de grande vulnerabilidade.

Neste sentido, os quatro municípios estudados formam um conjunto de experiências em que se busca reforçar a atuação do setor público na condução da implementação e consolidação da Estratégia de Saúde da Família, preservando a participação do Estado na prestação dos serviços visà-vis outros modelos de gestão, que implicam a transferência da gestão dos serviços para a iniciativa privada e a terceirização da força de trabalho.

Em relação aos nós críticos identificados na gestão de recursos humanos nesses municípios e que merecem reflexão mais aprofundada sobre estratégias de enfrentamento, estão a adesão das categorias médica edeenfermagem esuas respectivas representações ao novo modelo assistencial que orienta a Estratégia de Saúde da Família.

Concluindo, todas as iniciativas apresentadas procuram contribuir de alguma forma para aumentar a credibilidade dos profissionais da atenção primária à saúde, superar relações hierárquicas e 0 isolamento entre atenção primária e especializada, fundamentais para a reorganização dos serviços assistenciais em consonância com os princípios do SUS.

\section{Colaboradores}

MH M M endonça, M IC Martins e L Giovanella participaram igualmente de todas as etapas de elaboração do artigo. S Escorel participou da coordenação da pesquisa, da discussão dos resultados e aprovou a versão apresentada.

\section{Agradecimentos}

A pesquisa de campo foi realizada com 0 apoio da empresa de consultoria DM \&P - Tecnologia Gerencial em Desenvolvimento de Mercados e Produtos, com sedeem Belo H orizonte (M G). As pesquisadoras Junia M arçal eGiselleSilva deCarvalho, coordenadora técnica do trabalho de campo e responsável pela elaboração do banco de dados, respectivamente, contribuíram especialmente para a análise descritiva e discussão preliminar dos resultados apresentados neste artigo. 


\section{Referências}

1. Nogueira RP. Problemas de gestão e regulação do trabalho no SUS. [site da Internet] [acessado 2008 out 07]. Disponível em: http://www.observarh.org.br/ observarh/repertorio/Repertorio_ObservaRH / NESP-UnB/Problemas_gestao_regulacao.pdf

2. Cardoso JR. M acroeconomia e mercado de trabalho no Brasil: trajetória recente e tendência aparente. [site da Internet] [acessado 2008 out 07]. Disponível em: http://www.observarh.org.br/nesp/upload/arquivos/20060901045936_macroeconom_trab.pdf

3. Escorel S, Giovanellä L, M endonça $M H M, M$ agaIhães R, Senna M CM. Avaliação da implantação do programa saúde da família em dez grandes centros urbanos: síntese dos principais resultados. Brasília: Ministério da Saúde; 2002.

4. Mendonça MHM. O SUS e política de recursos humanos em Saúde. Divulgação em Saúde Pública para Debate 2000; 40:13-16.

5. Dedecca CS, Rosandiski EM, Carvalho MS, Barbie re CV. Dimensão do Setor de Atendimento à Saúde no Brasil. [site da Internet] [acessado 2008 out 07]. Disponível em: http://www.abep.nepo.unicamp.br/ site_eventos_abep/PDF/ABEP2004_426.pdf

6. Site da Internet. Disponível em: $\bar{h}$ ttp://www.saude gov.br

7. Site da Internet. Disponível em: http://www. obsnetims.org.br/pesquisa - relatório 2008

8. Yin RK. Estudo de caso: planejamento emétodos. Porto Alegre: Bookman; 2005.

9. Giovanella L, M endonça MHM, Escorel S, Almeida PF, Senna MCM, Fausto MCR, Delgado M, Andrade CLT, Martins MIC, Cunha MS, Pacheco C. Estudo de caso sobre implementação da Estratégia de Saúde da Família em Florianópolis (SC) [relatório de pesquisa]. Rio de Janeiro: Nupes/DAPS/ENSP/Fiocruz; 2009.

10. Saltman RB, Rico A, Boerma WGW. Primary care in the driver's seat? Organizational reform in European primary care. Berkshire: Open University Press; 2006

11. Giovanella L, M endonça M HM, Escorel S, Almeida PF, Senna MCM, Fausto MCR, Delgado M, Andrade CLT, Martins MIC, Cunha MS, Pacheco C. Estudo de caso sobre implementação da Estratégia de Saúde da Família em Belo H orizonte. [relatório de pesquisa]. Rio de Janeiro: Nupes/DAPS/EN SP/Fiocruz; 2009.

12. Giovanella $L$, M endonça $M H M$, Escorel $S$, Almeida PF, Senna MCM, Fausto MCR, Delgado M, Andrade CLT, Martins MIC, Cunha MS, Pacheco C. Estudo de caso sobre implementação da Estratégia de Saúde da Família em Aracaju (SE). [relatório de pesquisa]. Rio de Janeiro: Nupes/DAPS/ENSP/Fiocruz; 2009.
13. Giovanella $L, M$ endonça $M H M$, Escorel $S$, Almeida PF, Senna MCM, Fausto MCR, Delgado M, Andrade CLT, Martins MIC, Cunha MS, Pacheco C. Estudo de caso sobre implementação da Estratégia de Saúde da Família em Vitória (ES). [relatório de pesquisa]. Rio de Janeiro: Nupes/DAPS/ENSP/Fiocruz; 2009.

14. Machado MH, Rego S, Teixeira M. Os males da especialização médica no Brasil, Ciência Hoje 1997; 22(130):22-30.

15. Seixas PH, Stella RCR. M édicos e mercado de trabalho: experiências de interiorização, estímulos e alternativas de fixação. In: Negri B, Faria R, Viana ALd'A, organizadores. Recursos humanos em Saúde: política, desenvolvimento e mercado de trabalho. Campinas: Unicamp; 2002. p. 354-371.

16. Padilha RQ. PROMED: Programa de Incentivo a Mudanças Curriculares nas Escolas M édicas. In: Brasil. Ministério da Saúde. Políticas de recursos humanos em saúde. Brasília: Ministério da Saúde; 2002.

17. Gugliemi MC. A Política Pública "Saúde da Família" e a Permanência - Fixação do Profissional em M edicina: um estudo de campo em Pernambuco [tese]. Rio de Janeiro (RJ): IMS, Universidade Estadual do Rio de Janeiro; 2006.

18. Pierantoni CR, Varella TC, França T. Recursos humanos e gestão do trabalho em Saúde: da teoria à prática. In: Barros AFR, organizador. Observatório de Recursos Humanos em Saúde no Brasil: estudos e análises. Brasília: Ministério da Saúde; 2004.

19. Souza HM. Pólos de capacitação, formação e educação permanente para o programa saúde da família. In: Brasil. M inistério da Saúde. Políticas de Recursos Humanos em Saúde. Brasília: Ministério da Saúde; 2002.

Artigo apresentado em 26/04/2010

Aprovado em 07/06/2010

Versão final apresentada em 14/06/2010 